\title{
Short-time focused ultrasound hyperthermia enhances liposomal doxorubicin delivery and antitumor efficacy for brain metastasis of breast cancer
}

\author{
This article was published in the following Dove Press journal: \\ International Journal of Nanomedicine \\ 19 September 2014 \\ Number of times this article has been viewed
}

\author{
Sheng-Kai Wu' \\ Chi-Feng Chiang' \\ Yu-Hone Hsu ${ }^{1,4}$ \\ Tzu-Hung Lin ${ }^{2}$ \\ Houng-Chi Liou ${ }^{2}$ \\ Wen-Mei Fu ${ }^{2}$ \\ Win-Li Lin ${ }^{1,3}$
}

'Institute of Biomedical Engineering, College of Medicine and College of Engineering, ${ }^{2}$ Institute of Pharmacology, College of Medicine, National Taiwan University, Taipei, Taiwan; ${ }^{I}$ Institute of Biomedical Engineering and Nanomedicine, National Health Research Institutes, Miaoli, Taiwan; ${ }^{4}$ Department of Neurosurgery, Cheng-Hsin General Hospital, Taipei, Taiwan
Correspondence: Win-Li Lin Institute of Biomedical Engineering, College of Medicine and College of Engineering, National Taiwan University, No I, Sec I, Jen-Ai Road,

Taipei, Taiwan 100

Tel +886 223।2 3456 ext $8 \mid 450$

Fax +886223940049

Email winli@ntu.edu.tw

Wen-Mei Fu

Pharmacological Institute,

College of Medicine, National Taiwan

University, No I, Sec I, Jen-Ai Road,

Taipei, Taiwan 100

Tel +886 223123456 ext 88319

Fax +886 2232 I II 27

Email wenmei@ntu.edu.tw
Abstract: The blood-brain/tumor barrier inhibits the uptake and accumulation of chemotherapeutic drugs. Hyperthermia can enhance the delivery of chemotherapeutic agent into tumors. In this study, we investigated the effects of short-time focused ultrasound (FUS) hyperthermia on the delivery and therapeutic efficacy of pegylated liposomal doxorubicin (PLD) for brain metastasis of breast cancer. Murine breast cancer 4T1-luc2 cells expressing firefly luciferase were injected into female BALB/c mice striatum tissues and used as a brain metastasis model. The mice were intravenously injected with PLD $(5 \mathrm{mg} / \mathrm{kg})$ with/without 10-minute transcranial FUS hyperthermia on day 6 after tumor implantation. The amounts of doxorubicin accumulated in the normal brain tissues and tumor tissues with/without FUS hyperthermia were measured using fluorometry. The tumor growth for the control, hyperthermia, PLD, and PLD + hyperthermia groups was measured using an IVIS spectrum system every other day from day 3 to day 11. Cell apoptosis and tumor characteristics were assessed using immunohistochemistry. Short-time FUS hyperthermia was able to significantly enhance the PLD delivery into brain tumors. The tumor growth was effectively inhibited by a single treatment of PLD + hyperthermia compared with both PLD alone and short-time FUS hyperthermia alone. Immunohistochemical examination further demonstrated the therapeutic efficacy of PLD plus short-time FUS hyperthermia for brain metastasis of breast cancer. The application of short-time FUS hyperthermia after nanodrug injection may be an effective approach to enhance nanodrug delivery and improve the treatment of metastatic cancers.

Keywords: hyperthermia, focused ultrasound (FUS), pegylated liposomal doxorubicin (PLD), brain metastasis of breast cancer

\section{Introduction}

An estimated $40 \%$ of cancer patients develop brain metastasis, which is a major cause of mortality and morbidity. ${ }^{1}$ Brain metastasis is prevalent in breast cancer, lung cancer, and melanoma. The standard management of patients with brain metastasis has been optimized over time, owing to technical improvements in surgery and radiation therapy. 2,3 Chemotherapy with cytotoxic drugs is limited to palliation, as the efficacy depends on the chemosensitivity of the primary tumor. ${ }^{4}$ Multiple strategies have been developed with limited success to improve brain metastasis chemotherapy, including combination therapy with radiation, novel chemotherapeutics formulations, direct administration of chemotherapeutics, and targeted vascular disruption. ${ }^{5,6}$ One of the main reasons for the failure of brain chemotherapy is the presence of the blood-brain barrier (BBB), which is located at the brain vascular endothelium. ${ }^{7}$ The BBB is frequently impaired in brain 
metastasis, creating the blood-brain tumor barrier (BTB), with many brain metastases displaying elevated vascular permeability. ${ }^{8}$ Although the BTB had perceptible permeability changes in most brain metastases, it does not necessarily indicate an absence of barrier function. For example, the average vascular permeability of brain metastasis of breast cancer is less than $15 \%$ that of peripheral breast tumors. ${ }^{9,10}$

Therapeutic hyperthermia is a procedure for heating tumor tissues to an effective temperature for a desired time. Mild hyperthermia is used in the clinic to improve the outcome of chemotherapy or radiotherapy without significant side effects. ${ }^{11,12}$ Hyperthermia can improve the antitumor effect of some chemotherapeutic compounds, such as liposomal doxorubicin. The mechanisms of this treatment include increased perfusion as well as increased vascular permeability and interstitial microconvection. Hence, hyperthermia enhances the delivery of therapeutic agents and improves tissue oxygenation. ${ }^{13,14}$ In mice, treatment with temperature-sensitive liposomal doxorubicin and local hyperthermia results in higher intratumor drug concentrations and improves therapeutic efficacy, compared with treatment with either free doxorubicin or liposomal doxorubicin without hyperthermia. ${ }^{15}$

Current heating strategies include local, regional, and whole-body hyperthermia, which can be implemented by microwave, radiofrequency, laser, and ultrasound. Ultrasound hyperthermia can be achieved noninvasively with focused ultrasound transducers. High-intensity focused ultrasound (HIFU) is employed to study the release and delivery of temperature-sensitive liposomes in animal models. HIFU hyperthermia can produce a high concentration of doxorubicin in the targeted region. ${ }^{16,17}$

The goals of this study are to investigate the feasibility of using short-time focused ultrasound (FUS) hyperthermia to enhance the delivery of nanodrugs into metastatic brain tumors and assess the therapeutic efficacy in a small animal model.

\section{Materials and methods}

All experimental protocols were approved by the Institutional Animal Care and Use Committee of the Medical College, National Taiwan University.

\section{Preparations of cells and the intracranial tumor model}

Murine 4T1-luc2 breast cancer cells (ATCC ${ }^{\circledR}$ CRL-2539TM) expressing firefly luciferase (luc2 vector) were cultured in Dulbecco's Modified Eagle's Medium (DMEM) supplemented with $10 \%$ heat-inactivated fetal bovine serum, penicillin
$(100 \mathrm{U} / \mathrm{mL}) /$ streptomycin $(100 \mu \mathrm{g} / \mathrm{mL})$ in $10 \mathrm{~cm}$ tissue culture plates in a $5 \% \mathrm{CO}_{2}$-containing incubator at $37^{\circ} \mathrm{C}$. Cell number and viability were determined using a hemocytometer and trypan blue exclusion.

Eight-week-old female BALB/c mice were used in this study. The mice were housed with a 12-hour light/dark cycle and allowed free access to water and standard diet. During the tumor implantation, the mice were anesthetized by exposure to $1 \%$ to $3 \%$ isoflurane. A total of $2 \times 10^{4}$ of $4 \mathrm{~T} 1$-luc 2 tumor cells suspended in $2 \mu \mathrm{L}$ of phosphate buffered saline (PBS) were slowly injected into the right caudate putamen $0.5 \mathrm{~mm}$ anterior and $2.0 \mathrm{~mm}$ lateral to the bregma at a depth of $3 \mathrm{~mm}$ from the dura over a 3-minute duration. The needle was left in place for 5 minutes and then withdrawn slowly. The scalp wound was closed with 6-0 polydioxanone suture.

\section{Focused ultrasound (FUS) system and short-time FUS hyperthermia}

Ultrasound sonication was generated by a $500 \mathrm{kHz}$, singleelement focused transducer (H104MR; Sonic Concepts, Bothell, WA, USA) with a diameter of $64 \mathrm{~mm}$ and a curvature radius of $62.64 \mathrm{~mm}$. The half-maximum pressure amplitude diameter and length of the focal zone were $3 \mathrm{~mm}$ and $8 \mathrm{~mm}$, respectively. The radiofrequency signal was supplied by a function generator (33120A; Agilent, Palo Alto, CA, USA) whose output was amplified by a radiofrequency power amplifier (75A250A; Amplifier Research, Souderton, PA, USA). The acoustic beam was transmitted to the brain directly by a removable cone replete with degassed water. The FUS was precisely targeted using a stereotaxic apparatus (Stoelting, Wood Dale, IL, USA). The center of the focal spot was approximately $3 \mathrm{~mm}$ below the cone tip. The transducer was applied with a continuous wave of $500 \mathrm{kHz}$.

To determine the FUS parameters used in this study, we first tested different power levels and measured the temperature responses at the focal zone during short-time FUS hyperthermia. A T-type thermocouple (5TC-TT-T-36-36; Omega Engineering, Stamford, Connecticut, USA) was inserted into the brain tissue of the anesthetized mouse $(n=3)$ to monitor the temperature response of the focal point before, during, and after FUS hyperthermia (Figure 1A). The thermocouple was connected to a data acquisition system (TC-2190; National Instruments, Austin, Texas, USA). Figure 1B shows a typical temperature response at the focal point measured with a thermocouple at a power level of 2.2 Watt. When the power of the FUS transducer was turned on, the temperature at the focal point rapidly rose over $40^{\circ} \mathrm{C}$ and then approached a steady-state value of approximately $42^{\circ} \mathrm{C}-43^{\circ} \mathrm{C}$. 

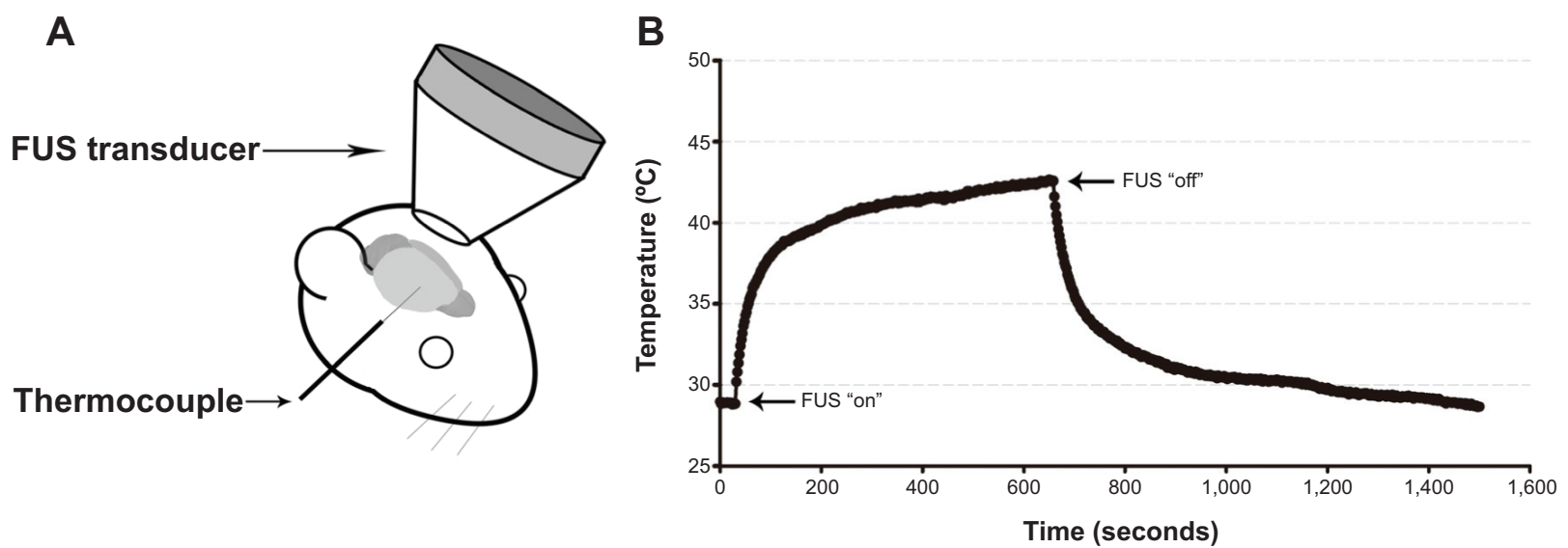

Figure I (A) Schematic representation of the experimental setup for temperature measurement. (B) Temporal temperature response at the focal point in the mouse brain before, during and after 10 minutes of continuous FUS sonication.

Notes: The brain temperature of an anesthetized mouse was approximately $29^{\circ} \mathrm{C}$ before the FUS. The arrows in (B) indicate the time for FUS "on" and "off."

Abbreviation: FUS, focused ultrasound.

After 10 minutes of sonication, the power was turned off to allow the temperature to gradually return to the baseline. This set of FUS parameters (power level of 2.2 Watt, sonication duration of 10 minutes, and maximum pressure at the focal point of $0.97 \mathrm{MPa}$ ) was applied to the following FUS hyperthermia experiments without inserting a thermocouple.

\section{Experimental grouping}

The experiments included two parts: part 1, quantification of pegylated liposomal doxorubicin (PLD) in normal brain and tumor tissues (Figure 2A); and part 2, tumor growth responses to various treatment conditions (Figure 2B). To quantify the amount of PLD deposited in the normal brain and tumor tissues, we used 20 mice randomly divided into four groups: 1) normal brain without FUS hyperthermia $(\mathrm{n}=5), 2)$ normal brain with FUS hyperthermia $(\mathrm{n}=5)$, 3) brain tumor without FUS hyperthermia $(\mathrm{n}=5)$, and 4) brain tumor with FUS hyperthermia ( $\mathrm{n}=5)$. The PLD was injected as a bolus $(5 \mathrm{mg} / \mathrm{kg})$ approximately 1 minute before FUS hyperthermia. To examine the treatment efficacy, we used 24 mice randomly divided into four groups: 1) control ( $\mathrm{n}=6)$,

2) FUS hyperthermia alone ( $n=6), 3$ ) PLD alone ( $n=6)$, and 4) PLD + FUS hyperthermia ( $n=6)$. A total of 44 mice were used for the PLD quantification and treatment studies.

\section{Quantification of PLD entering the brain tissue}

To determine whether doxorubicin delivery was enhanced by short-time FUS hyperthermia, a dose of $5 \mathrm{mg} / \mathrm{kg}$ PLD (TTY Biopharm, Taipei, Taiwan) was administered

\section{A}

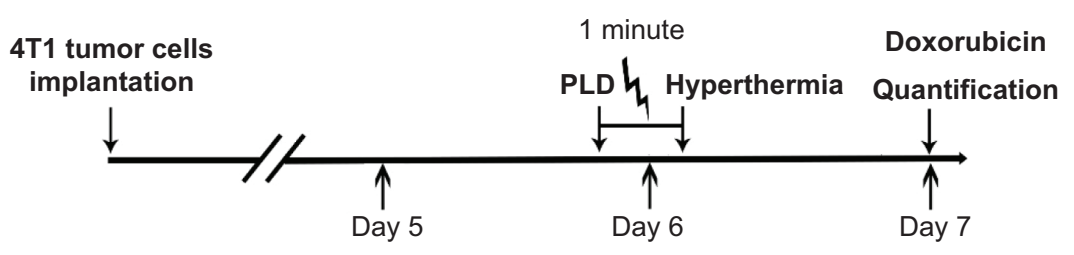

B

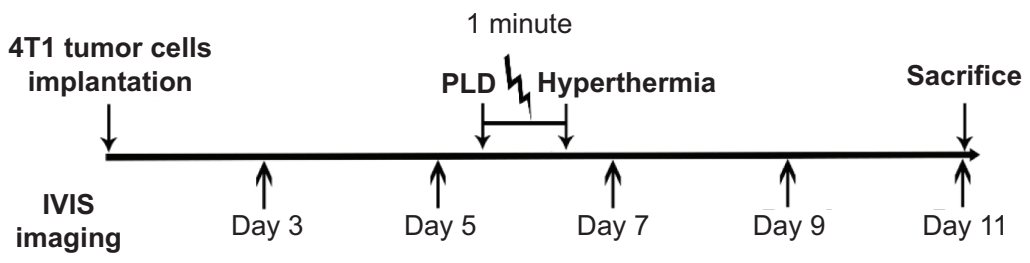

Figure 2 (A) Schedule for PLD injection and quantification. (B) Time course of tumor implantation and PLD and/or short-time FUS hyperthermia administration. Abbreviations: PLD, pegylated liposomal doxorubicin; FUS, focused ultrasound. 
through the tail vein on day 6 after tumor implantation with or without FUS hyperthermia (Figure 2A). To flush the PLD in the cerebral vessels, the brain was perfused via a transcardial method with normal saline 24 hours after the PLD administration. The amounts of doxorubicin deposited in tissues were then quantified using a method similar to Mayer et al. ${ }^{18}$ Briefly, the tissues were homogenized with $100 \mu \mathrm{L}$ of $10 \%(\mathrm{v} / \mathrm{v})$ Triton X-100, which lysed the liposomes and released the entrapped doxorubicin. Samples of the homogenate $(100 \mu \mathrm{L})$ were then placed in $2 \mathrm{~mL}$ microcentrifuge tubes, and $50 \mu \mathrm{L}$ of $10 \%(\mathrm{v} / \mathrm{v})$ Triton $\mathrm{X}-100,100 \mu \mathrm{L}$ of water, and $750 \mu \mathrm{L}$ of acidified isopropanol $(0.75 \mathrm{~N} \mathrm{HCl})$ were added. The tubes were vortexed to ensure complete mixing, and the doxorubicin was extracted overnight at $-20^{\circ} \mathrm{C}$. The next day, the microcentrifuge tubes were warmed to room temperature, vortexed for 5 minutes, and centrifuged at 15,000 $\mathrm{g}$ for 20 minutes. To correct for background fluorescence, the samples were compared with standard curve data from the fluorescence emission of known amounts of doxorubicin added to acidified isopropanol extracts of homogenized tumor tissue from untreated mice. The concentration of doxorubicin was measured using a fluorometer (excitation at $470 \mathrm{~nm}$ and emission at $590 \mathrm{~nm}$ ) (SpectraMax M2; Molecular Devices, Sunnyvale, CA, USA).

\section{Measurement of tumor growth by in vivo imaging}

Prior to in vivo imaging, the mice were anesthetized with isoflurane. D-luciferin (Gold Biotechnology Inc., St Louis, MO, USA) solution was then intraperitoneally injected $(150 \mathrm{mg} / \mathrm{kg})$. The mice were then imaged with an IVIS $^{\circledR}$ Spectrum, and bioluminescent signals were quantified using Living Image 3.0 (Caliper Life Sciences, Alameda, CA, USA). Images were taken every other day starting from day 3 and continuing up to day 11 after tumor implantation (Figure 2B). The PLD/hyperthermia treatment was performed on day 6 after tumor implantation when the measured bioluminescent value reached about approximately $10^{6}$ photons/seconds.

\section{Histology and immunohistochemistry}

Histological and immunohistochemical staining were performed on day 11 after tumor implantation. The mice were sacrificed, perfused with saline, and fixed with phosphate buffer containing 4\% paraformaldehyde. The brains were removed, postfixed with $4 \%$ paraformaldehyde at $4^{\circ} \mathrm{C}$ overnight, and then stored in a $30 \%$ sucrose solution at $4^{\circ} \mathrm{C}$ for 2 days. The brains were consecutively sliced to a thickness of $20 \mu \mathrm{m}$. At least three slices for the maximal tumor area were taken as the representative slices for each staining. Brain tissues were stained with hematoxylin-eosin (H\&E) for gross histological examination. For immunohistochemical analysis, tissue slices were pretreated with 3\% hydrogen peroxide to block endogenous peroxidase activity before incubation with a primary antibody. After blocking for 1 hour in $4 \%$ nonfat milk containing $1 \%$ Triton $\mathrm{X}-100$, the brain tissue slices were incubated overnight at $4{ }^{\circ} \mathrm{C}$ with a $\mathrm{Ki} 67$ primary antibody (1:200; Novus Biologicals, Littleton, CO, USA) in PBS. After a brief wash, the brain tissue slices were incubated with a horse antimouse biotinylated secondary antibody and processed with an avidin-biotin complex system (ABC kit; Vector Laboratories, Burlingame, CA, USA), which was visualized by incubating with $0.5 \%$ diaminobenzidine and $0.01 \%$ hydrogen peroxide in PBS. Finally, the brain tissue slices were washed in PBS and mounted on slides. All slices were microscopically evaluated by a pathologist in a blinded manner (Axio Imager A1; Carl Zeiss Ltd., Oberkochen, Germany). Digital images were analyzed using AxioVision software (Carl Zeiss Ltd., Oberkochen, Germany). Ki67positive cells and TUNEL-positive nuclei were counted in three different fields from three separate sections at a magnification of $\times 100$, with data presented as the percentage of the total number of tumor cells.

\section{TUNEL assay}

Tumor sections were processed for the TUNEL assay using a DeadEnd Fluorometric TUNEL system (Promega, Madison, WI, USA) following the manufacturer's instructions. Briefly, the slides were fixed with $4 \%$ formaldehyde and permeabilized with $20 \mu \mathrm{g} / \mathrm{mL}$ proteinase $\mathrm{K}$ and $0.2 \%$ Triton $\mathrm{X}-100$ in PBS. The slides were then labeled with a $\mathrm{TdT}$ reaction mixture for 60 minutes at $37^{\circ} \mathrm{C}$ and mounted with a mounting solution containing 4',6-diamidino-2-phenylindole (DAPI) (Sigma, St Louis, MO, USA). Fluorescence images of apoptotic cells (green) and cell nuclei (blue) were obtained using a confocal microscope (Axio Imager A1; Carl Zeiss Ltd., Oberkochen, Germany) with the fluorescein isothiocyanateDAPI setting (excitation at 340-380 $\mathrm{nm}$ and emission at 435-485 nm). All images were captured using the same exposure time. The pictures were merged using AxioVision Rel. 4.8 software (Carl Zeiss Ltd., Oberkochen, Germany).

\section{Statistical analysis}

All values are expressed as the mean \pm SEM (standard error of the mean). The results were analyzed with one-way analysis of variance with the post hoc Dunnet test. Statistical significance was defined as $P<0.05$. Calculations were 
performed on a computer using SPSS version 20.0 (SPSS Inc., Chicago, Illinois, USA).

\section{Results}

\section{Enhancement of PLD delivery to normal brain and tumor tissues by FUS hyperthermia}

Fluorometry was used to measure the doxorubicin from the PLD deposited in the tumor tissues and normal brain tissues for the groups with/without short-time FUS hyperthermia. Figure 3 shows the concentration of doxorubicin for tumor and normal brain tissues 24 hours after PLD administration, with five mice for each group. The concentration of doxorubicin was significantly greater in tumors treated with PLD + hyperthermia than in tumors treated with PLD alone. With the FUS sonication after PLD injection, the doxorubicin concentration in tumors was 2.4-fold that of the PLD group. In addition, Figure 3 also shows that the concentration of doxorubicin was significantly higher in tumors than in normal brain tissues in response to PLD injection alone.

\section{Short-time FUS hyperthermia enhances the cytotoxic action of PLD in tumors}

To evaluate the effect of short-time FUS hyperthermia on the antitumor action of PLD, tumor-bearing mice were treated a single time with different protocols on day 6 after tumor implantation. Tumor progressions were then evaluated by IVIS imaging every other day starting from day 3. Figure 4A

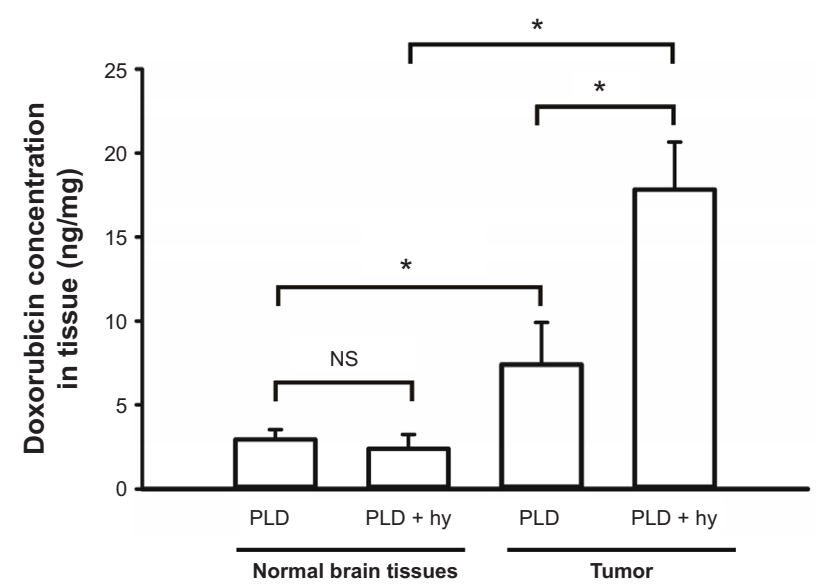

Figure 3 Doxorubicin concentrations in tumor and normal brain tissues in mice with and without short-time FUS hy.

Notes: Female BALB/c mice were transcranially implanted with 4TI-luc2 murine breast cancer cells and received $5 \mathrm{mg} / \mathrm{kg}$ PLD with or without transcranial FUS hyperthermia on day 6 after tumor implantation. FUS hyperthermia enhanced the PLD delivery in the tumor regions. Data are presented as the mean \pm SEM ( $n=5$ for each group). $* P<0.05$.

Abbreviations: FUS, focused ultrasound; PLD, pegylated liposomal doxorubicin; hy, hyperthermia; SEM, standard error of the mean; ns, not significant. shows the representative images of the bioluminescent signals, indicating that the photons increased exponentially in the control group. In addition, the signal pattern for the hyperthermia group treated with short-time FUS sonication alone was similar to the control group. The bioluminescent signal of the PLD alone group displayed slight inhibition. However, inhibition was markedly increased by additional treatment with short-time FUS hyperthermia following PLD injection. Figure 4B shows the results of the bioluminescent response, which indicate that the tumors treated with PLD + FUS hyperthermia were markedly inhibited on day 9 and day 11 compared with the control, FUS hyperthermia alone, or PLD groups. There were no significant differences among the latter three groups.

\section{Inhibition of proliferation in tumors by FUS hyperthermia plus PLD}

Four mice from each group (control, hyperthermia, PLD, and PLD + hyperthermia) were euthanized on day 11 after tumor implantation to obtain their brain tissues. The brain tissues were stained with H\&E. The tumor outlines could be clearly observed (Figure 5, top panel). The H\&E staining showed that the tumor size of the PLD + hyperthermia group was significantly smaller than the other three groups, which indicated that treatment with PLD plus short-time FUS hyperthermia could inhibit tumor growth. To examine the effect of various treatments on tumor cell proliferation, Ki67 immunohistochemistry was performed in the tumor tissues. As shown in the representative images (Figure 5, second panel), abundant Ki67-positive nuclei were observed in the tumors of the control and hyperthermia groups, while there was comparatively less Ki67 expression in the PLD and PLD + hyperthermia groups. The PLD + hyperthermia group had the lowest percentage of Ki67-positive cells (Figure 6A).

\section{Enhancement of TUNEL staining by} short-time FUS hyperthermia plus PLD

To further assess the antitumor effect, TUNEL staining was performed to analyze the apoptotic cells in the tumors. Figure 7 shows that there was no obvious tumor cell apoptosis in the control and hyperthermia groups, while TUNEL-positive tumor cells were prominent in the PLD + hyperthermia group. Treatment with PLD + hyperthermia resulted in a significant increase in apoptotic cell death (Figure 6B).

\section{Discussion}

In this study, we investigated the synergistic effect of shorttime FUS hyperthermia with an anticancer nanodrug on the 
A

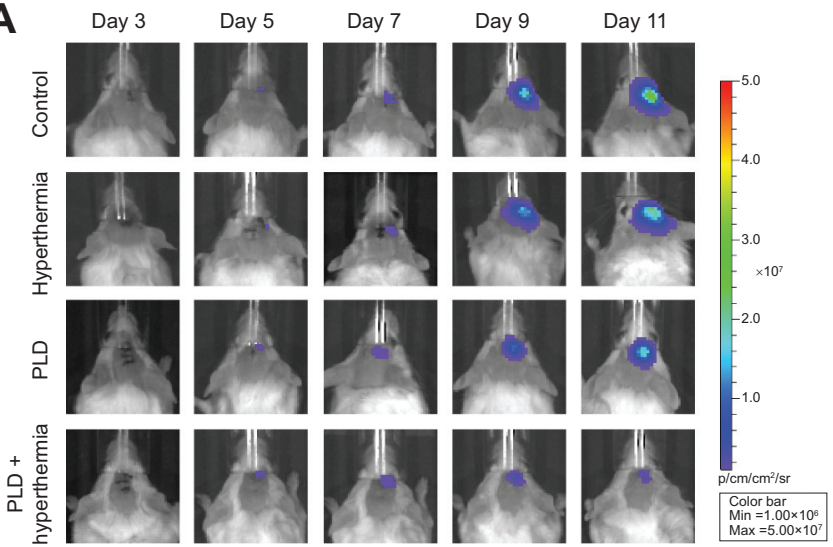

B

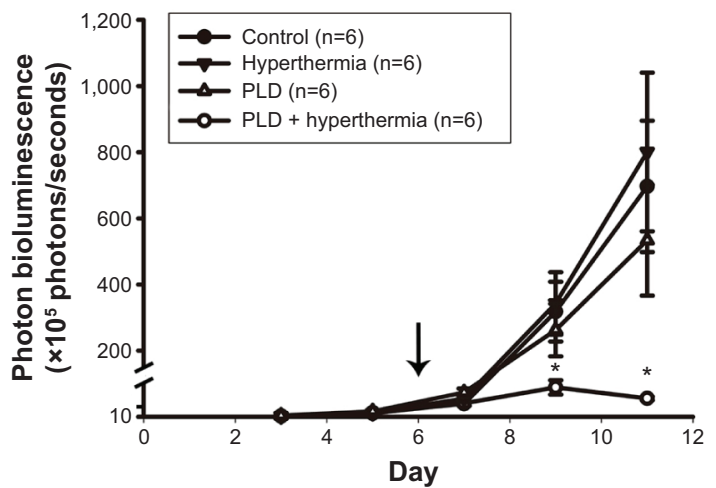

Figure 4 IVIS imaging of mouse brain tumors from day 3 to day II after tumor implantation.

Notes: (A) Mice with luciferase-expressing 4TI-luc2 breast cancer cells implanted in their striatum tissue. There were four groups: control (no treatment), hyperthermia, PLD, and PLD + hyperthermia. Treatment was executed on day 6 after tumor implantation, and images were obtained every other day. (B) Bioluminescent signals were quantified using the IVIS imaging system. The arrow indicates when the different treatments were performed. Note that PLD + hyperthermia markedly inhibited breast tumor growth in the brain. Data are presented as the mean \pm SEM ( $n=6$ for each group). $* P<0.05$ compared with the control group.

Abbreviations: PLD, pegylated liposomal doxorubicin; SEM, standard error of the mean.

treatment of brain metastasis of breast cancer. The application of short-time FUS hyperthermia in the tumor region produced a significant increase in doxorubicin accumulation in the brain tumors treated with systemic PLD (Figure 3). Furthermore, FUS hyperthermia enhanced the cytotoxicity of PLD, thereby inhibiting tumor growth (Figure 4). Figure 3 also shows that the application of short-time FUS hyperthermia in normal brain tissues does not increase the delivery of PLD into normal brain tissues. The elimination half-life of the PLD used in this study was 20-30 hours. ${ }^{19}$ Hong et al ${ }^{20}$ demonstrated that the drug concentration in tumors peaks at approximately 24 hours after injection of PLD and then decreases slowly over time. Based on this information, we chose 24 hours after treatment as the time point to quantify the concentration of PLD in normal brain and tumor tissues.

The transport of the nanodrug and its concentration distribution in tumor tissues are related to 1) the injected dosage; 2) the nanodrug concentration difference between the intravascular and extravascular regions; 3 ) the vascular density, vascular permeability, and interstitial diffusivity of the nanodrug; and 4) the fluid flow convection between the tumor tissues and the adjacent normal tissues. It has been shown that the application of hyperthermia can lead to increased blood flow and enhanced vascular permeability in tumors. ${ }^{14,21}$ A previous study showed that the extravasation of nanoparticles (100 nm liposomes) in tumor tissues
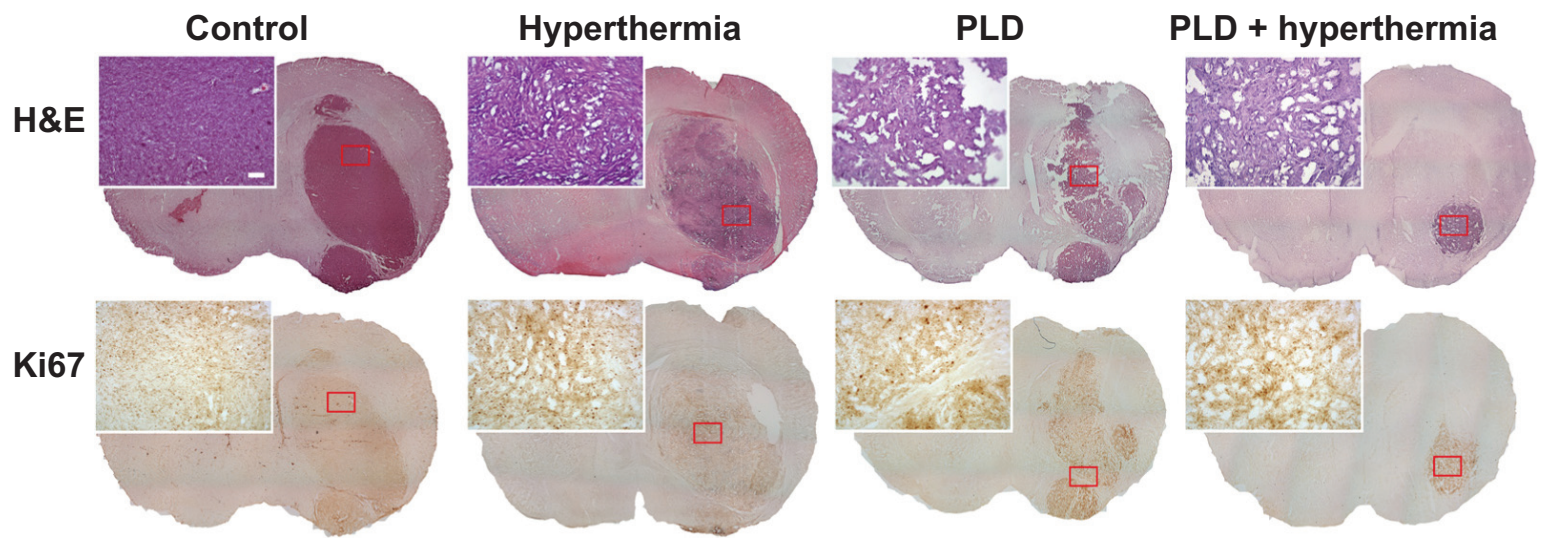

Figure 5 Histological (H\&E) and immunohistochemical (Ki67) staining performed in tumor regions following the different treatments.

Notes: Mice were implanted with 4TI-luc2 tumor cells with treatment performed on day 6 . The mice were sacrificed on day II. Tumor slices were then obtained for staining. Tumors treated with PLD + hyperthermia had smaller tumors than the control group based on H\&E staining (upper panels). Ki67 expression was associated with cell proliferation. Mild Ki67 expression was found in the tumor area of the PLD + hyperthermia-treated group. Scale bars $=100 \mu \mathrm{m}$ and I mm, respectively.

Abbreviations: PLD, pegylated liposomal doxorubicin; H\&E, hematoxylin and eosin. 

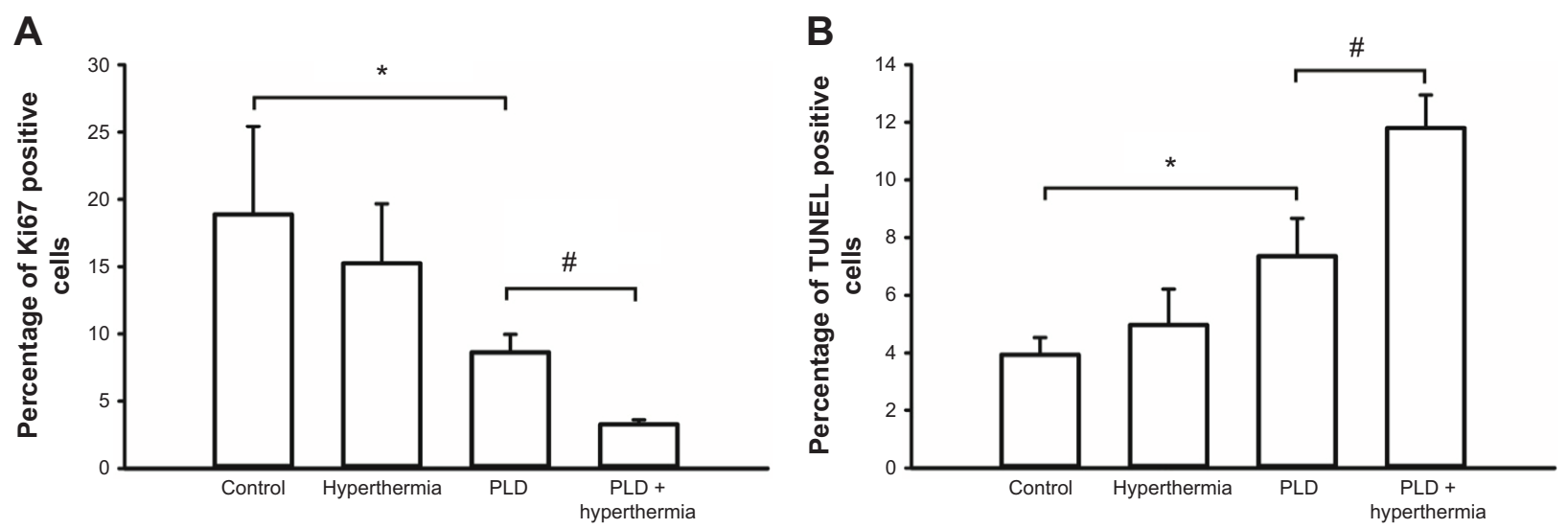

Figure 6 Quantitative analysis of Ki67-positive and TUNEL-positive cells.

Notes: (A) The number of Ki67 positive cells significantly decreased in the PLD + hyperthermia group compared with those in the PLD group. (B) TUNEL-positive cells were increased in the PLD + hyperthermia group. Data are shown as the mean \pm SEM ( $n=3$ for each group). ${ }^{* P}<0.05$ compared with the control group. ${ }^{* P}<0.05$ compared with the PLD group.

Abbreviations: PLD, pegylated liposomal doxorubicin; TUNEL, terminal deoxynucleotidyl transferase dUTP-mediated nick-end labeling; SEM, standard error of the mean; dUTP, deoxyuridine triphosphate.

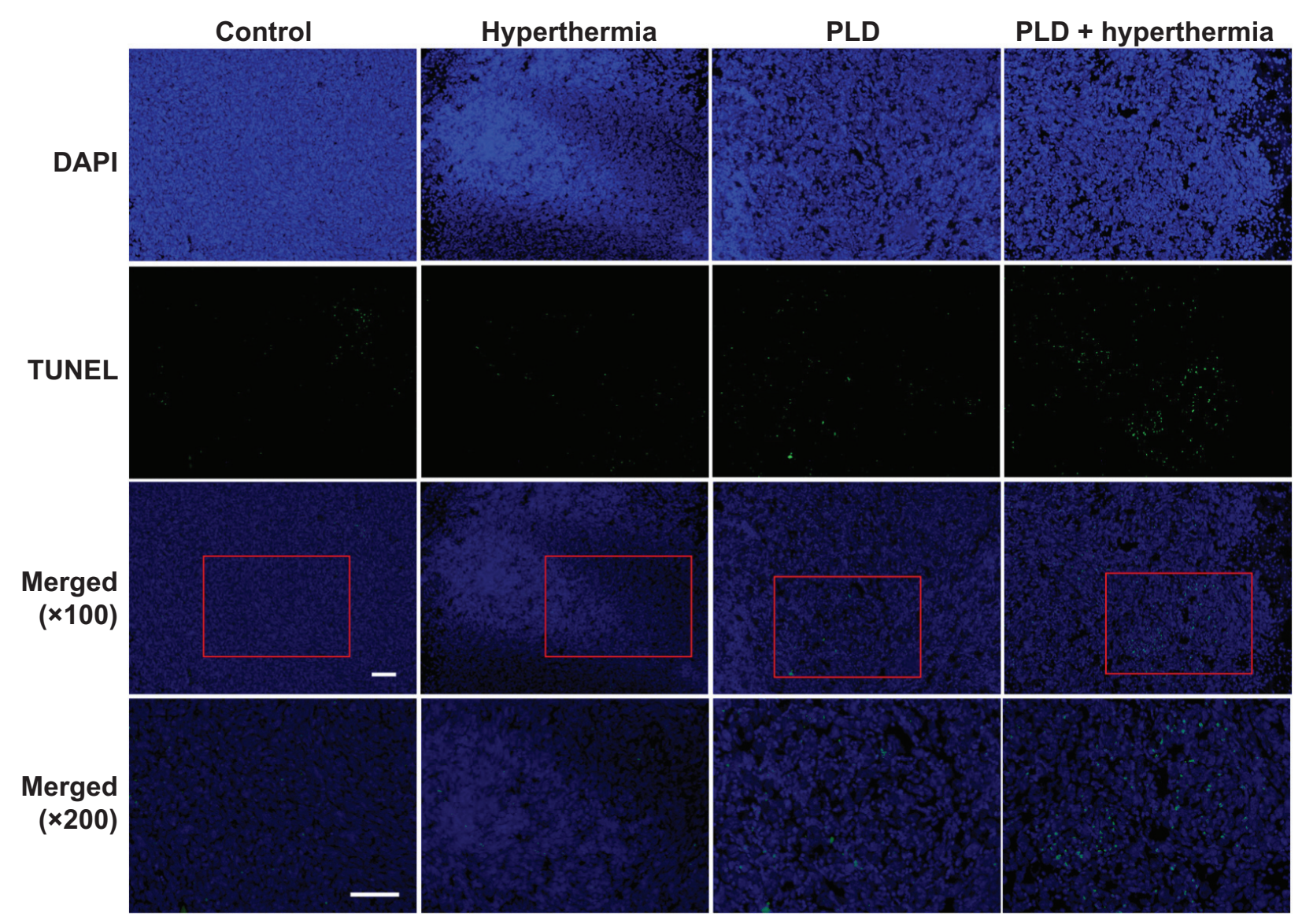

Figure 7 TUNEL staining used to detect apoptotic cells in the tumor.

Notes: Mice were implanted with 4TI-luc2 tumor cells with treatment performed on day 6. The mice were sacrificed on day II. Tumor slices were then obtained for staining. In the first row, tissues were stained with DAPI to visualize the tumor cells (blue) in the brain section. In the second row, tissues were stained with terminal TUNEL (green). The third and fourth rows display an overlay of the two images showing the distribution of apoptotic cells in the tumors at different magnifications. Strong apoptotic signals were expressed in the tumors treated with PLD + hyperthermia. Scale bar $=100 \mu \mathrm{m}$.

Abbreviations: PLD, pegylated liposomal doxorubicin; DAPI, 4',6-diamidino-2-phenylindole; TUNEL, terminal deoxynucleotidyl transferase dUTP-mediated nick-end labeling; dUTP, deoxyuridine triphosphate. 
increased with temperatures $40^{\circ} \mathrm{C}$ to $42^{\circ} \mathrm{C}$, approaching maximal extravasation at $42^{\circ} \mathrm{C} .{ }^{22} \mathrm{~A}$ significant enhancement of doxorubicin accumulation was shown in tumors treated with PLD compared with normal tissues due to enhanced permeability and retention effects in the tumors. ${ }^{23}$

In this study, we employed transcranial FUS for shorttime hyperthermia following intravenous injection of PLD for the treatment of brain metastasis of breast cancer. The application of FUS hyperthermia following PLD injection can significantly increase the delivery of PLD into brain tumor tissues, thereby inhibiting tumor growth. Although the use of chemotherapeutic agents for brain metastasis of cancer is usually limited by the $\mathrm{BBB} / \mathrm{BTB},{ }^{8}$ we are the first to demonstrate that short-time FUS hyperthermia may be a feasible technique for improving the treatment efficacy of anticancer nanodrugs on brain metastasis. In addition, we showed that the application of short-time FUS hyperthermia in normal brain tissues did not increase the delivery of PLD into normal brain tissues. This indicates that the application of short-time FUS hyperthermia does not change the integrity of the intact BBB. However, liposomal drugs display increased cellular uptake and reduced efflux through ATP-binding cassette (ABC) transporters. ${ }^{24}$ This would lead to a low accumulation of doxorubicin in normal brain tissues 24 hours after PLD injection.

A similar study has been conducted in a peripheral murine mammary adenocarcinoma model treated with liposomal doxorubicin plus pulsed-HIFU. ${ }^{25}$ There were no significant differences in the delivery of liposomal doxorubicin in this model. The sonication time and temperature increase in their pulsed-HIFU were 2 minutes and $41^{\circ} \mathrm{C}-42^{\circ} \mathrm{C}$, respectively, at a doxorubicin dosage of $2 \mathrm{mg} / \mathrm{kg}$. In our study, we employed a 10 minutes FUS hyperthermia and a dosage of $5 \mathrm{mg} / \mathrm{kg}$ doxorubicin to treat brain tumors. An enhancement of drug delivery and antitumor effect was demonstrated in our study. The discrepancy between our study and the previous study may stem from the different tumor models and sonication times used. A higher vascular permeability with a shorter sonication time may be the reason the other study did not produce a significant enhancement in nanodrug delivery.

We demonstrated the feasibility of using transcranial short-time FUS hyperthermia with an anticancer nanodrug to treat breast cancer brain metastasis. We applied a single treatment for a small tumor (day 6 after tumor implantation). Early stage tumors possess high vascular density with relatively intact vascular barriers, hampering the transport of PLD into tumor tissues. ${ }^{26}$ Our results show that a 10 minutes FUS hyperthermia treatment can increase BTB permeability and blood perfusion of the tumors, thereby enhancing the transport of nanodrugs into interstitial tumor tissues to hinder tumor growth. For further investigation, a relatively large tumor should be selected with multiple sonication locations to enhance the delivery of nanodrugs over the entire tumor. Recently, thermosensitive liposomal doxorubicin has been reported for hyperthermia-related chemotherapy. ${ }^{27-30}$ The therapeutic efficacy of short-time FUS hyperthermia may be improved if this type of thermosensitive liposomal doxorubicin is taken into consideration.

It has been previously reported that FUS with microbubbles, which are ultrasound contrast agents that are in clinical use, can disrupt the local BBB/BTB to enhance the delivery of various types of therapeutic agents into brain tumors. ${ }^{31-36}$ However, the interaction between microbubbles and FUS can produce unnecessary side effects, such as erythrocyte extravasations, intracerebral microhemorrhages, edema, and inflammation. ${ }^{37-39}$ Moreover, therapeutic agents will accumulate in normal brain tissues leading to serious side effects when the normal brain tissues are sonicated. Side effects of conventional hyperthermia are cerebral necrosis, edema, focal hemorrhage, and infarction when inadequate exposure to heat is applied. It has been demonstrated that thermally induced BBB opening in normal brain tissue is always accompanied by brain tissue damage. The thermal dose threshold for BBB opening is estimated to be 12.3 minutes in $\mathrm{CEM}_{43}$ (cumulative equivalent minutes at $43^{\circ} \mathrm{C}$ ). ${ }^{40}$ In this study, we applied a thermal dose much less than the threshold value to avoid damage to normal brain tissues. Furthermore, we demonstrate that short-time FUS hyperthermia can enhance the delivery of PLD into the sonicated brain tumor tissues without increasing drug deposition in sonicated normal brain tissues. Overall, this is the first study showing the feasibility of transcranial short-time FUS hyperthermia to enhance drug delivery and chemotherapy for brain tumors without damaging normal brain tissues.

The most common side effects for PLD treatment are palmar-plantar erythrodysesthesia, stomatitis, skin reaction, and hematological toxicity. The side effects are associated with dose and pharmacokinetic parameters such as Cmax (peak plasma concentration), AUC (area under concentration $\times$ time curve), and elimination. ${ }^{41}$ In this study, a significant inhibition to the tumor growth was achieved using $5 \mathrm{mg} / \mathrm{kg}$ (equivalent to approximately $15 \mathrm{mg} / \mathrm{m}^{2}$ in clinic) $)^{42}$ PLD with FUS hyperthermia. For clinical treatment, a dose of $48-60 \mathrm{mg} / \mathrm{m}^{2}$ is used every 4 weeks for the treatment of recurrent/resistant breast carcinoma to optimize clinical efficacy and to minimize the occurrence of dose schedule-related adverse 
events. ${ }^{41}$ The dosage of PLD used in this study was much lower than that used in the clinic and could alleviate the side effects of PLD. With the application of FUS hyperthermia after PLD injection, the doxorubicin concentration in tumors was 2.4-fold higher than PLD alone. Hence, the combination of PLD and short-time FUS hyperthermia can achieve therapeutic efficacy while reducing the side effects of PLD.

On day 11, animals of the control and hyperthermia groups displayed an inability to take food and move. The PLD group showed a slight inability to move, and the PLD + hyperthermia group appeared normal. Therefore, we sacrificed all animals on day 11 and did not show the survival data in this study. However, it is reasonable to predict that animals with smaller brain tumor and better conditions tend to have longer survival times. Survival observation and large tumor treatment will be the topics in our future studies.

The main antitumor action of doxorubicin is believed to be DNA damage due to topoisomerase II inhibition, which induces cancer cell apoptosis. ${ }^{43}$ In addition, exposure to FUS sonication and high temperature would increase drug delivery into cancer cells and enhance their chemosensitivity. ${ }^{44-46}$ Apoptosis was observed using the TUNEL technique in different treatment groups. As shown in Figure 7, TUNELpositive (apoptotic) cells were detected in the PLD alone group, and many more apoptotic cells appeared in the PLD + hyperthermia group. This may be the reason that a combination of PLD and short-time FUS hyperthermia can significantly inhibit tumor growth.

\section{Conclusion}

In this study, we demonstrated that short-time FUS hyperthermia enhances anticancer nanodrug delivery and antitumor effects for the treatment of metastatic tumors in a mouse model. Short-time FUS hyperthermia significantly improved the delivery of liposomal doxorubicin into tumor tissues when sonication was applied locally in the brain tumor regions. However, drug delivery into the normal brain tissues was not affected by hyperthermia. This technique has potential for clinical translation when combined with nanodrugs under magnetic resonance imaging guidance and monitoring.

\section{Acknowledgments}

This work was supported by research grants from the National Science Council of Taiwan (NSC 101-2221-E-002-015MY3) and the National Health Research Institutes of Taiwan (NHRI ME-100-PP-13).

\section{Disclosure}

The authors report no conflicts of interest in this work.

\section{References}

1. Nayak L, Lee EQ, Wen PY. Epidemiology of brain metastases. Curr Oncol Rep. 2012;14(1):48-54.

2. Barnholtz-Sloan JS, Yu C, Sloan AE, et al. A nomogram for individualized estimation of survival among patients with brain metastasis. Neuro Oncol. 2012;14(7):910-918.

3. Sperduto PW, Chao ST, Sneed PK, et al. Diagnosis-specific prognostic factors, indexes, and treatment outcomes for patients with newly diagnosed brain metastases: a multi-institutional analysis of 4,259 patients. Int J Radiat Oncol Biol Phys. 2010;77(3):655-661.

4. Soffietti R, Ruda R, Trevisan E. Brain metastases: current management and new developments. Curr Opin Oncol. 2008;20(6):676-684.

5. Gabathuler R. Blood-brain barrier transport of drugs for the treatment of brain diseases. CNS Neurol Disord Drug Targets. 2009;8(3): 195-204.

6. Peereboom DM. Chemotherapy in brain metastases. Neurosurgery. 2005;57(5 Suppl):S54-S65; discusssion S51-S54.

7. Deeken JF, Loscher W. The blood-brain barrier and cancer: transporters, treatment, and Trojan horses. Clin Cancer Res. 2007;13(6): $1663-1674$.

8. Gerstner ER, Fine RL. Increased permeability of the blood-brain barrier to chemotherapy in metastatic brain tumors: establishing a treatment paradigm. J Clin Oncol. 2007;25(16):2306-2312.

9. Hasegawa H, Ushio Y, Hayakawa T, Yamada K, Mogami H. Changes of the blood-brain barrier in experimental metastatic brain tumors. $J$ Neurosurg. 1983;59(2):304-310.

10. Lockman PR, Mittapalli RK, Taskar KS, et al. Heterogeneous bloodtumor barrier permeability determines drug efficacy in experimental brain metastases of breast cancer. Clin Cancer Res. 2010;16(23): 5664-5678.

11. Issels RD, Lindner LH, Verweij J, et al; European Organisation for Research and Treatment of Cancer Soft Tissue and Bone Sarcoma Group (EORTC-STBSG); European Society for Hyperthermic Oncology (ESHO). Neo-adjuvant chemotherapy alone or with regional hyperthermia for localised high-risk soft-tissue sarcoma: a randomised phase 3 multicentre study. Lancet Oncol. 2010;11(6):561-570.

12. van der Zee J, Gonzalez Gonzalez D, van Rhoon GC, van Dijk JD, van Putten WL, Hart AA. Comparison of radiotherapy alone with radiotherapy plus hyperthermia in locally advanced pelvic tumours: a prospective, randomised, multicentre trial. Lancet. 2000;355(9210):1119-1125.

13. Song CW. Effect of local hyperthermia on blood flow and microenvironment: a review. Cancer Res. 1984;44(10 Suppl):4721s-4730s.

14. Song CW, Park HJ, Lee CK, Griffin R. Implications of increased tumor blood flow and oxygenation caused by mild temperature hyperthermia in tumor treatment. Int J Hyperthermia. 2005;21(8):761-767.

15. Kong G, Anyarambhatla G, Petros WP, et al. Efficacy of liposomes and hyperthermia in a human tumor xenograft model: importance of triggered drug release. Cancer Res. 2000;60(24):6950-6957.

16. Gasselhuber A, Dreher MR, Partanen A, et al. Targeted drug delivery by high intensity focused ultrasound mediated hyperthermia combined with temperature-sensitive liposomes: computational modelling and preliminary in vivovalidation. Int J Hyperthermia. 2012;28(4):337-348.

17. Partanen A, Yarmolenko PS, Viitala A, et al. Mild hyperthermia with magnetic resonance-guided high-intensity focused ultrasound for applications in drug delivery. Int $J$ Hyperthermia. 2012;28(4): 320-336.

18. Mayer LD, Dougherty G, Harasym TO, Bally MB. The role of tumorassociated macrophages in the delivery of liposomal doxorubicin to solid murine fibrosarcoma tumors. J Pharmacol Exp Ther. 1997; 280(3):1406-1414.

19. Gabizon A, Shmeeda H, Barenholz Y. Pharmacokinetics of pegylated liposomal Doxorubicin: review of animal and human studies. Clin Pharmacokinet. 2003;42(5):419-436. 
20. Hong RL, Huang CJ, Tseng YL, et al. Direct comparison of liposomal doxorubicin with or without polyethylene glycol coating in C-26 tumorbearing mice: is surface coating with polyethylene glycol beneficial? Clin Cancer Res. 1999;5(11):3645-3652.

21. Kong G, Dewhirst MW. Hyperthermia and liposomes. Int $J$ Hyperthermia. 1999;15(5):345-370.

22. Kong G, Braun RD, Dewhirst MW. Characterization of the effect of hyperthermia on nanoparticle extravasation from tumor vasculature. Cancer Res. 2001;61(7):3027-3032.

23. Maeda $\mathrm{H}$. Macromolecular therapeutics in cancer treatment: the EPR effect and beyond. J Control Release. 2012;164(2):138-144.

24. Pinzon-Daza ML, Campia I, Kopecka J, Garzon R, Ghigo D, Riganti C. Nanoparticle- and liposome-carried drugs: new strategies for active targeting and drug delivery across blood-brain barrier. Curr Drug Metab. 2013;14(6):625-640.

25. Frenkel V, Etherington A, Greene M, et al. Delivery of liposomal doxorubicin (Doxil) in a breast cancer tumor model: investigation of potential enhancement by pulsed-high intensity focused ultrasound exposure. Acad Radiol. 2006;13(4):469-479.

26. Lin CY, Tseng HC, Shiu HR, Wu MF, Chou CY, Lin WL. Ultrasound sonication with microbubbles disrupts blood vessels and enhances tumor treatments of anticancer nanodrug. Int $J$ Nanomedicine. 2012;7:2143-2152.

27. Chen D, Sun K, Mu H, et al. $\mathrm{pH}$ and temperature dual-sensitive liposome gel based on novel cleavable mPEG-Hz-CHEMS polymeric vaginal delivery system. Int J Nanomedicine. 2012;7:2621-2630.

28. Li L, ten Hagen TL, Haeri A, et al. A novel two-step mild hyperthermia for advanced liposomal chemotherapy. J Control Release. 2014;174: 202-208.

29. Li L, ten Hagen TL, Hossann M, et al. Mild hyperthermia triggered doxorubicin release from optimized stealth thermosensitive liposomes improves intratumoral drug delivery and efficacy. $J$ Control Release. 2013;168(2):142-150

30. Yang Y, Yang Y, Xie X, et al. PEGylated liposomes with NGR ligand and heat-activable cell-penetrating peptide-doxorubicin conjugate for tumor-specific therapy. Biomaterials. 2014;35(14):4368-4381.

31. Liu HL, Hua MY, Yang HW, et al. Magnetic resonance monitoring of focused ultrasound/magnetic nanoparticle targeting delivery of therapeutic agents to the brain. Proc Nat Acad Sci U S A. 2010; 107(34):15205-15210.

32. Park EJ, Zhang YZ, Vykhodtseva N, McDannold N. Ultrasound-mediated blood-brain/blood-tumor barrier disruption improves outcomes with trastuzumab in a breast cancer brain metastasis model. $J$ Control Release. 2012;163(3):277-284.

33. Treat LH, McDannold N, Zhang Y, Vykhodtseva N, Hynynen K. Improved anti-tumor effect of liposomal doxorubicin after targeted blood-brain barrier disruption by MRI-guided focused ultrasound in rat glioma. Ultrasound Med Biol. 2012;38(10):1716-1725.

34. Yang FY, Wong TT, Teng MC, et al. Focused ultrasound and interleukin-4 receptor-targeted liposomal doxorubicin for enhanced targeted drug delivery and antitumor effect in glioblastoma multiforme. J Control Release. 2012;160(3):652-658.
35. Park J, Zhang Y, Vykhodtseva N, Jolesz FA, McDannold NJ. The kinetics of blood-brain barrier permeability and targeted doxorubicin delivery into brain induced by focused ultrasound. J Control Release. 2012;162(1):134-142.

36. Kovacs Z, Werner B, Rassi A, Sass JO, Martin-Fiori E, Bernasconi M. Prolonged survival upon ultrasound-enhanced doxorubicin delivery in two syngenic glioblastoma mouse models. J Control Release. 2014;187C:74-82.

37. Hynynen K, McDannold N, Sheikov NA, Jolesz FA, Vykhodtseva N. Local and reversible blood-brain barrier disruption by noninvasive focused ultrasound at frequencies suitable for trans-skull sonications. NeuroImage. 2005;24(1):12-20.

38. Liu HL, Wai YY, Chen WS, et al. Hemorrhage detection during focused-ultrasound induced blood-brain barrier opening by using susceptibility-weighted magnetic resonance imaging. Ultrasound Med Biol. 2008;34(4):598-606.

39. McDannold N, Vykhodtseva N, Raymond S, Jolesz FA, Hynynen K. MRI-guided targeted blood-brain barrier disruption with focused ultrasound: histological findings in rabbits. Ultrasound Med Biol. 2005;31(11):1527-1537.

40. McDannold N, Vykhodtseva N, Jolesz FA, Hynynen K. MRI investigation of the threshold for thermally induced blood-brain barrier disruption and brain tissue damage in the rabbit brain. Magn Reson Med. 2004;51(5):913-923.

41. Lyass O, Uziely B, Ben-Yosef R, et al. Correlation of toxicity with pharmacokinetics of pegylated liposomal doxorubicin (Doxil) in metastatic breast carcinoma. Cancer. 2000;89(5):1037-1047.

42. Office of New Drugs, Center for Drug Evaluation and Research, Food and Drug Administration. Guidance for Industry: Estimating the Maximum Safe Starting Dose in Initial Clinical Trials for Therapeutics in Adult Healthy Volunteers. Rockville, MD: Center for Drug Evaluation and Research, Food and Drug Administration; 2005.

43. Hurley LH. DNA and its associated processes as targets for cancer therapy. Nat Rev Cancer. 2002;2(3):188-200.

44. Akiyoshi T, Wada T, Arinaga S, Koba F, Tsuji H. Enhanced chemosensitivity of cells from malignant effusions under condition of exposure to high temperature. Jpn J Surg. 1986;16(5):323-329.

45. Crowder KC, Hughes MS, Marsh JN, et al. Sonic activation of molecularly-targeted nanoparticles accelerates transmembrane lipid delivery to cancer cells through contact-mediated mechanisms: implications for enhanced local drug delivery. Ultrasound Med Biol. 2005; 31(12):1693-1700.

46. Mohan P, Rapoport N. Doxorubicin as a molecular nanotheranostic agent: effect of doxorubicin encapsulation in micelles or nanoemulsions on the ultrasound-mediated intracellular delivery and nuclear trafficking. Mol Pharm. 2010;7(6):1959-1973.
International Journal of Nanomedicine

\section{Publish your work in this journal}

The International Journal of Nanomedicine is an international, peerreviewed journal focusing on the application of nanotechnology in diagnostics, therapeutics, and drug delivery systems throughout the biomedical field. This journal is indexed on PubMed Central, MedLine, CAS, SciSearch ${ }^{\circledR}$, Current Contents ${ }^{\circledR} /$ Clinical Medicine,
Dovepress

Journal Citation Reports/Science Edition, EMBase, Scopus and the Elsevier Bibliographic databases. The manuscript management system is completely online and includes a very quick and fair peer-review system, which is all easy to use. Visit http://www.dovepress.com/ testimonials.php to read real quotes from published authors. 